PROCEEDINGS OF THE

AMERICAN MATHEMATICAL SOCIETY

Volume 134, Number 8, Pages 2281-2283

S 0002-9939(06)08232-3

Article electronically published on February 2, 2006

\title{
A COUNTEREXAMPLE CONCERNING LINE-FREE GROUPS AND COMPLETE ERDŐS SPACE
}

\author{
JAN J. DIJKSTRA AND JAN VAN MILL
}

(Communicated by N. Tomczak-Jaegermann)

\begin{abstract}
We present a weakly closed, one-dimensional, line-free subgroup of the separable Banach space $c$ that is not homeomorphic to complete Erdôs space. The existence of this example disproves a conjecture of Dobrowolski, Grabowski, and Kawamura.
\end{abstract}

Complete Erdős space was first featured by Erdős in [8, who proved that it is totally disconnected and one-dimensional. It can be represented by, for instance,

$$
\mathfrak{E}_{\mathrm{c}}=\left\{z \in \ell^{2}: z_{i} \in \mathbb{R} \backslash \mathbb{Q} \text { for each } i \in \mathbb{N}\right\},
$$

where $\ell^{2}$ is the Hilbert space of square summable real sequences. $\mathfrak{E}_{\mathrm{c}}$ is a universal element of the class of almost zero-dimensional spaces; for background information see [11, 9, 3, 4, 5]. A subset of a topological space is called a $C$-set if it can be written as an intersection of clopen subsets of the space. A topological space is called almost zero-dimensional if every point has a neighbourhood basis consisting of C-sets. Every almost zero-dimensional space is at most one-dimensional; see [11, 10, 1.

An additive subgroup of a vector space is called line-free if it does not contain nontrivial linear subspaces. It is remarked in [2] that a topological classification of the line-free closed subgroups of Banach spaces produces a classification of all closed subgroups of Banach spaces. Let $G$ be an arbitrary nondiscrete, weakly closed, line-free, additive subgroup of a separable Banach space $E$. Dobrowolski, Grabowski, and Kawamura 7] proved that $G$ is homeomorphic to complete Erdős space whenever $E$ is reflexive. In addition, Ancel, Dobrowolski, and Grabowski 2] showed that $E$ contains zero-dimensional examples of such groups $G$ precisely if $E$ contains an isomorphic copy of $c_{0}$. These results prompted Dobrowolski, Grabowski, and Kawamura 7] to formulate the following

Conjecture. Every separable, nondiscrete, weakly closed, one-dimensional, linefree subgroup of a Banach space is homeomorphic to $\mathfrak{E}_{\mathrm{c}}$.

We present a counterexample to this conjecture, thereby finding a new topological type that closed subgroups of Banach spaces can have. We shall distinguish our example from $\mathfrak{E}_{\mathrm{c}}$ by the following property of $\mathfrak{E}_{\mathrm{c}}$. A topological space is called somewhere zero-dimensional if it contains a point at which the space is

Received by the editors December 8, 2004 and, in revised form, February 25, 2005.

2000 Mathematics Subject Classification. Primary 46B25, 54F45.

Key words and phrases. Banach space, line-free group, weakly closed, complete Erdős space, almost zero-dimensional.

(C)2006 American Mathematical Society Reverts to public domain 28 years from publication 
zero-dimensional, that is, the point has a clopen neighbourhood basis. Dijkstra, van Mill, and Steprāns [6] have shown that $\mathfrak{E}_{\mathrm{c}}$ has the property that every point $x \in \mathfrak{E}_{\mathrm{c}}$ has a neighbourhood $U$ such that every closed subset of $U$ is either empty or somewhere zero-dimensional.

Counterexample. We construct our counterexample in the Banach space $c$. We find it convenient to represent $c$ as the space of all continuous real-valued functions $f$ on the convergent sequence $\{0\} \cup\{1 / n: n \in \mathbb{N}\}$. The norm $\|f\|=\sup \{|f(1 / n)|$ : $n \in \mathbb{N}\}$ makes $c$ a separable Banach space. For $n \in \mathbb{N}$ let $\varphi_{n}$ be the element of the dual of $c$ that is given by $\varphi_{n}(f)=2^{n} f(1 / n)$. Since $\left\{\varphi_{n}: n \in \mathbb{N}\right\}$ is clearly a total sequence of functionals, we have that

$$
G=\left\{f \in c: \varphi_{n}(f) \in \mathbb{Z} \text { for each } n \in \mathbb{N}\right\}
$$

is a line-free, weakly closed, additive subgroup of $c$. We first verify that $G$ is almost zero-dimensional and hence that $\operatorname{dim} G \leq 1$. Consider an arbitrary closed $\varepsilon$-ball $B_{\varepsilon}(f)=\{g \in c:\|g-f\| \leq \varepsilon\}$ in $c$. Let $g \in G \backslash B_{\varepsilon}(f)$ and note that $|g(1 / n)-f(1 / n)|>\varepsilon$ for some $n \in \mathbb{N}$. Then $\{h \in G: h(1 / n)=g(1 / n)\}$ is an obviously clopen subset of $G$ that is disjoint from $B_{\varepsilon}(f)$. Thus $G \cap B_{\varepsilon}(f)$ is a C-set in $G$, proving the almost zero-dimensionality of $G$. The fact $\operatorname{dim} G \leq 1$ also follows from [2, Theorem 3.1], when we note that the $\varphi_{n}$ 's form a norming sequence; see Dijkstra and van Mill [5, Remark 30].

We shall now show with the method of Erdös 8 that for each $\varepsilon>0$ the set $A=G \cap B_{\varepsilon}(\mathbf{0})$, where $\mathbf{0}$ stands for the zero function, is not zero-dimensional at each of its points. We may then conclude that $\operatorname{dim} G \geq 1$ and that $G$ is not homeomorphic to $\mathfrak{E}_{\mathrm{c}}$. (However, according to [4, Propositions 6.3 and 6.10] $G$ is homeomorphic to a dense subset of $\mathfrak{E}_{\mathrm{c}}$.) Let $f \in A$ be arbitrary. Since $A=-A$ we may assume that $f(0) \leq 0$. Let $U$ be a subset of $A \cap B_{\varepsilon / 3}(f)$ such that $f \in U$. We show that $U$ has boundary points in $A$. For each $n \in \mathbb{N}$ we let $\alpha_{n} \in G$ be defined by $\alpha_{n}(x)=2^{-n}$ for $x \leq 1 / n$ and $\alpha_{n}=0$ for $x>1 / n$. Note that $\left\|\alpha_{n}\right\|=\alpha_{n}(0)=2^{-n}$. We construct by recursion a sequence $g_{1}, g_{2}, g_{3}, \ldots$ in $U$ as follows. We put $g_{1}=f$. Assume that $g_{n-1}$ has been found. Since $U$ is bounded and $g_{n-1} \in U$, there is a $k \in\{0\} \cup \mathbb{N}$ such that

$$
g_{n-1}+k \alpha_{n} \in U \text { and } g_{n-1}+(k+1) \alpha_{n} \notin U .
$$

Defining $g_{n}=g_{n-1}+k \alpha_{n}$ we trivially have the following properties:

(1) $g_{n} \geq g_{n-1}$,

(2) $g_{n}+\alpha_{n} \in G \backslash U$, and

(3) $\left\|g_{n}-g_{n-1}\right\|=g_{n}(0)-g_{n-1}(0)$.

Since the sequence $g_{1}(0), g_{2}(0), \ldots$ is nondecreasing and bounded by $\varepsilon$, we have that it converges, say, to $L$. By property (3) we have $\sum_{n=1}^{\infty}\left\|g_{n+1}-g_{n}\right\|=L-g_{1}(0)$, thus $g_{1}, g_{2}, \ldots$ is a Cauchy sequence. Put $g=\lim _{n \rightarrow \infty} g_{n}$ and note that $g$ is in the closure of $U$ in $A$ because $A$ is closed. Since the closure of $U$ is contained in $B_{\varepsilon / 3}(f)$, we have $g(0) \leq f(0)+\varepsilon / 3 \leq \varepsilon / 3$. Select an $N \in \mathbb{N}$ such that $2^{-N}<\varepsilon / 3$ and $g(1 / n)<2 \varepsilon / 3$ for each $n>N$. Let $n>N$. If $i<n$, then $\left(g_{n}+\alpha_{n}\right)(1 / i)=$ $g_{n}(1 / i) \in[-\varepsilon, \varepsilon]$. If $i \geq n$, then

$$
-\varepsilon \leq g_{n}(1 / i) \leq\left(g_{n}+\alpha_{n}\right)(1 / i)=g_{n}(1 / i)+2^{-n} \leq g(1 / i)+\varepsilon / 3 \leq \varepsilon .
$$

Thus $g_{n}+\alpha_{n} \in A$ for every $n>N$. With property (2) we have that $g=$ $\lim _{n \rightarrow \infty}\left(g_{n}+\alpha_{n}\right)$ is also in the closure of $A \backslash U$, thus $U$ is not clopen in $A$ and $A$ is not zero-dimensional at $f$. 
Since $c$ is isomorphic to $c_{0}$, our construction also applies to that space and, by the Hahn-Banach Theorem, to every locally convex space that contains an isomorphic copy of $c_{0}$.

\section{REFERENCES}

[1] M. Abry and J. J. Dijkstra, On topological Kadec norms, Math. Ann. 332 (2005), 759-765. MR 2179775

[2] F. D. Ancel, T. Dobrowolski, and J. Grabowski, Closed subgroups in Banach spaces, Studia Math. 109 (1994), 277-290. MR.1274013 (95d:46009)

[3] J. J. Dijkstra and J. van Mill, Homeomorphism groups of manifolds and Erdös space, Electron. Res. Announc. Amer. Math. Soc. 10 (2004), 29-38. MR2048429 (2005c:57025)

[4] J. J. Dijkstra and J. van Mill, Erdös space and homeomorphism groups of manifolds, preprint.

[5] J. J. Dijkstra and J. van Mill, Characterizing complete Erdös space, preprint.

[6] J. J. Dijkstra, J. van Mill and J. Steprāns, Complete Erdős space is unstable, Math. Proc. Cambridge Philos. Soc. 137 (2004), 465-473. MR2092071

[7] T. Dobrowolski, J. Grabowski, and K. Kawamura, Topological type of weakly closed subgroups in Banach spaces, Studia Math. 118 (1996), 49-62. MR1373624 (97d:46013)

[8] P. Erdős, The dimension of the rational points in Hilbert space, Ann. of Math. 41 (1940), 734-736. MR0003191 (2:178a)

[9] K. Kawamura, L. G. Oversteegen, and E. D. Tymchatyn, On homogeneous totally disconnected 1-dimensional spaces, Fund. Math. 150 (1996), 97-112. MR.1391294 (97d:54060)

[10] M. Levin and R. Pol, A metric condition which implies dimension $\leq 1$, Proc. Amer. Math. Soc. 125 (1997), 269-273. MR1389528 (97e:54033)

[11] L. G. Oversteegen and E. D. Tymchatyn, On the dimension of certain totally disconnected spaces, Proc. Amer. Math. Soc. 122 (1994), 885-891. MR1273515 (95b:54040)

Faculteit der Exacte Wetenschappen/Afdeling Wiskunde, Vrije Universiteit, De Boelelaan $1081^{a}, 1081 \mathrm{HV}$ Amsterdam, The Netherlands

E-mail address: dijkstra@cs.vu.nl

Faculteit der Exacte Wetenschappen / Afdeling Wiskunde, Vrije Universiteit, De Boelelaan $1081^{a}, 1081 \mathrm{HV}$ Amsterdam, The Netherlands

E-mail address: vanmill@cs.vu.nl 